\title{
Sunitinib in the therapy of malignant paragangliomas: report on the efficacy in a SDHB mutation carrier and review of the literature
}

${ }^{1}$ Department of Experimental and Clinical Biomedical Sciences, University of Florence, Florence, Italy ${ }^{2}$ Department of Diagnostic Radiology 2, Azienda OspedalieraUniversitaria Careggi, Florence, Italy ${ }^{3}$ General and Surgical Unit, Azienda Ospedaliera-Universitaria Careggi, Florence, Italy ${ }^{4}$ Division of Nuclear Medicine, Azienda Ospedaliera-Universitaria Careggi, Florence, Italy ${ }^{5}$ Endocrinology Unit, Azienda Ospedaliera-Universitaria Careggi, Florence, Italy

Correspondence to: Massimo Mannelli

Department Experimental and Clinica Biomedical Sciences "Mario Serio", University of Florence

Viale Pieraccini 6

50139 - Florence, Italy

massimo.mannelli@unifi.it

Received on Mar/8/2016 Accepted on Jul/25/2016
Letizia Canu', Silvia Pradella², Elena Rapizzi', Rossella Fucci', Andrea Valeri ${ }^{3}$, Vittorio Briganti ${ }^{4}$, Valentino Giachè', Gabriele Parenti ${ }^{5}$, Tonino Ercolino', Massimo Mannelli'

\begin{abstract}
SUMMARY
Metastatic pheochromocytomas (PHEOs) and paragangliomas (sPGLs) are rare neural crest-derived tumors with a poor prognosis. About $50 \%$ of them are due to germ-line mutations of the $S D H B$ gene. At present, there is no cure for these tumors. Their therapy is palliative and represented by different options among which antiangiogenic drugs, like sunitinib, have been hypothesized to be effective especially in malignant $S D H B$ mutated tumors. We report the effects of sunitinib therapy in a SDHB mutation carrier affected by a malignant sPGL. During 101 weeks of therapy at different doses, sunitinib was able to cause a partial response and then a stable disease for a total of 78 weeks. This favorable response is the longest, out of the 35 so far reported in the literature, registered in a patient treated exclusively with sunitinib but, similarly to the other responses, the effect was limited in time. From our analysis of the scanty data present in the literature, the effect of sunitinib does not seem to be different among wild-type patients and those carrying a cluster $1 \mathrm{germ}$-line mutation. Sunitinib seems able to slow the disease progression in some patients with malignant PHEO/PGL and therefore may represent a therapeutic option, although randomized controlled studies are needed to assess its efficacy definitively in the treatment of these aggressive tumors. Arch Endocrinol Metab. 2017;61(1):90-7
\end{abstract}

\section{INTRODUCTION}

$\mathrm{P}$ heochromocytomas (PHEOs) and paragangliomas (PGLs) are neural crest-derived tumors (1). They are benign in about $90 \%$ of cases. Malignancy is diagnosed in the presence of metastases in organs devoid of chromaffin tissue such as bones, lymph nodes, liver and lungs.

Malignancy rate mostly depends on genetic background; about $50 \%$ of malignant PHEO/PGL are due to a germ-line mutation in the $S D H B$ gene (2-3).

Patients with benign PHEOs/sPGLs are cured by the surgical removal of the tumor while the treatment of malignant PHEOs/PGLs is palliative and aimed at prolonging patient survival and/or improving patient's quality of life (4). In the presence of a metastatic PHEO/PGL, 5 year survival is about 50\% (5-7).

Treatment of patients with malignant PHEOs/ sPGLs stems on several options: surgery, when feasible, is generally performed on the primary tumor and is mostly aimed at limiting the effects of high levels of catecholamines on target organs and, if radionuclide therapy is programmed, at enhancing $\mathrm{I}^{131}$-metaiodobenzylguanidine (MIBG) uptake by the remaining metastatic lesions (8). Radionuclide therapy using somatostatin analogs tracers has seldom proven to be effective (9). Chemotherapy has been employed mostly in progressive disease, with partial success, combining different drugs such as cyclophosphamide, vincristine and dacarbazine (CVD) (10) or with the alkylating drug temozolomide causing stable disease in up to $50 \%$ of the cases (11).

More recently other compounds have been proposed. Among these, drugs such as sunitinib have been hypothesized to be effective in $S D H B$ mutated PGL in view of their genetic profile, characterized by an activation of the angiogenic pathway $(12,13)$. 
In this paper, we report on the effect obtained by sunitinib, administered as monotherapy, in a $S D H B$ mutation carrier affected by a metastatic PGL and review the literature reporting the response to sunitinib in similar cases.

\section{CASE REPORT}

A 35 year old Caucasian male with a metastatic abdominal paraganglioma was referred to our Unit in September 2013. The patient, presenting a congenital right kidney hypoplasia, had already undergone surgery twice: at the age of 10 years, when a PGL localized near the left kidney was surgically removed, and at the age of 31 when he was operated for a local recurrence.

In 2012 he started presenting symptoms of catecholamine excess like hypertensive crises, palpitations and headache. At that time, urinary normetanephrine $(\mathrm{NMNu})$ was reported to be elevated. A ${ }^{18} \mathrm{FDG}$-PET showed persistent disease at the primary site and uptake in the left ischium. A I ${ }^{123}$ MIBG scintigraphy resulted positive only at the bone level. A ${ }^{111}$-In-Pentetrotide scan (Octreoscan) showed a low density of somatostatin receptors.

In September 2013, at admission, the laboratory tests showed a very high level of $\mathrm{NMNu}(8927$ $\mathrm{mcg} / 24 \mathrm{~h})$. A CT scan showed a new large abdominal recurrence $46 \times 49 \times 59 \mathrm{~mm}$ in size, located in the left lumbar-aortic region, other smaller abdominal peritoneal lesions (maximum diameter $20 \mathrm{~mm}$ ) as well as several liver metastases.

A ${ }^{99} \mathrm{Tc}$-diphosphonate bone scintigraphy resulted negative.

After written informed consent the patient underwent genetic testing, including all the major susceptibility genes. A heterozygous $\mathrm{G}>\mathrm{A}$ transversion variant at position $+\mathrm{l}$ of intron 4 was found in the $S D H B$ gene.

Despite doxazosine therapy at the dose of $2 \mathrm{mg} /$ day, the patient blood pressure resulted 140/105 $\mathrm{mmHg}$. Therefore, doxazosin dosage was progressively increased until normotension was obtained.

In October 2013, compression of the left ureter by the abdominal mass caused hydronephrosis and a sharp increase in serum creatinine $(2.40 \mathrm{mg} / \mathrm{dL})$. The obstruction was resolved by a pigtail insertion.

After two months (November 2013, t0), without any anticancer therapy, a CT scan showed a significant increase in size of the main lesion $(69 \times 56 \times 77 \mathrm{~mm})$ (Figure 1B). NMNu resulted further increased (10147 $\mathrm{mcg} / 24 \mathrm{~h}$ ) (Figure 2). In view of a disease progression (PD), the patient started Sunitinib therapy.
Disease progression was evaluated during the follow up by RECIST criteria (version 1.1) (14). The main abdominal recurrence was considered the target lesion.

Sunitinib doses and time schedules are reported in Figure 3. Both time schedules and doses were in time adjusted to the maximal time length and drug doses accepted by the patient, depending on the drug induced side effects (Table 1 ).

In February 2014 (tl), at first follow up, a partial response $(\mathrm{PR})$ as documented by a reduction in size of the main abdominal lesion $(37 \times 35 \times 36 \mathrm{~mm})$ and the other abdominal and liver metastases (Figure 1C), as well as a significant decrease in NMNu levels (5572 $\mathrm{mcg} / 24 \mathrm{~h}$ ) were found (Figure 2). The ${ }^{18}$ FDG-PET scan showed a reduction in the uptake of the liver metastases and in the number and uptake of the abdominal lesions. The uptake in the main abdominal lesion didn't change significantly (Figure 4B).

In July 2014 (t2), after additional 5 months of therapy, the disease was found stable at CT (main abdominal lesion: 37x38x36 mm) (SD) (Figure 1D) and ${ }^{18}$ FDG-PET (Figure 4C) while NMNu levels continued to decline $(3276 \mathrm{mcg} / 24 \mathrm{~h})$ (Figure 2).

At the third follow up in December 2014 (t3), the main abdominal lesion was further decreased in size $(27 \times 34 \times 34 \mathrm{~mm})$ while the other abdominal lesions and the liver metastases resulted unchanged (Figure 1E).

The ${ }^{18}$ FDG-PET scan showed a lower uptake of liver metastases and no change in the bone lesion (Figure 4D). The levels of $\mathrm{NMNu}$ were $1907 \mathrm{mcg} / 24 \mathrm{~h}$ (Figure 2). In May 2015 (t4), at the fourth follow up, the patient presented PD on CT (Figure IF) and ${ }^{18}$ FDG-PET (Figure 4E). The main abdominal lesion had increased in size $(51 \times 38 \times 60 \mathrm{~mm})$ and two new peritoneal lesions appeared while the liver metastases resulted unchanged. According to these findings, $\mathrm{NMNu}$ was found increased to $9250 \mathrm{mcg} / 24 \mathrm{~h}$ (Figure 2).

In October 2015 (t5), after almost two years of therapy with Sunitinib, we observed a further increase in the size $(62 \times 50 \times 75 \mathrm{~mm})$ of the main abdominal lesion and in the number of liver metastases (Figure 1G) lately confirmed by ${ }^{18}$ FDG-PET (Figure 4F).

To date the patient has completed 101 weeks of therapy and has PD, according to the RECIST criteria 1.1. He's continuing Sunitinib therapy at low doses ( $25 \mathrm{mg}$ /day 2 weeks on, l week off) with limited side effects. Radiosurgery on the primary tumor and the liver and bone metastatic lesions is ongoing. 


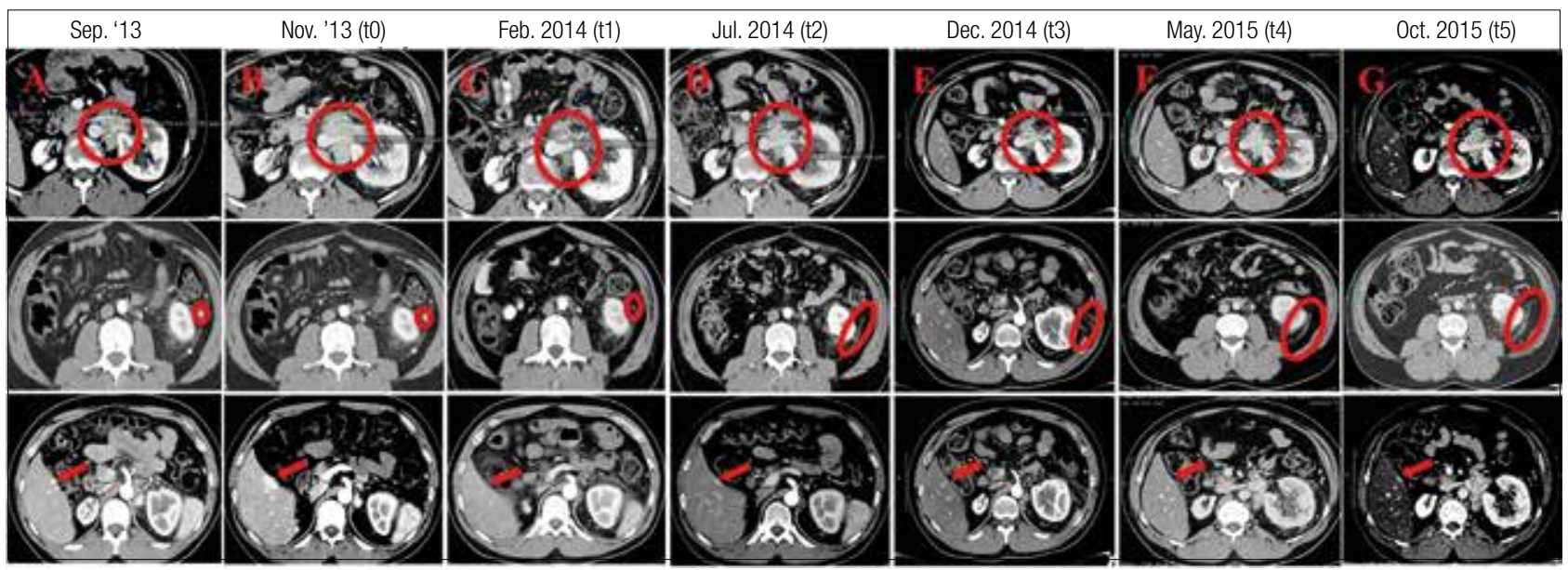

Figure 1. CT scan before sunitinib therapy: Sept. '13 (A) and Nov.'13 (B), and during the follow up: t1 (C), t2 (D), t3 (E), t4 (F) and t5 (G). In the first line the main abdominal lesion, in the second line another abdominal lesion and in the third line liver metastases.

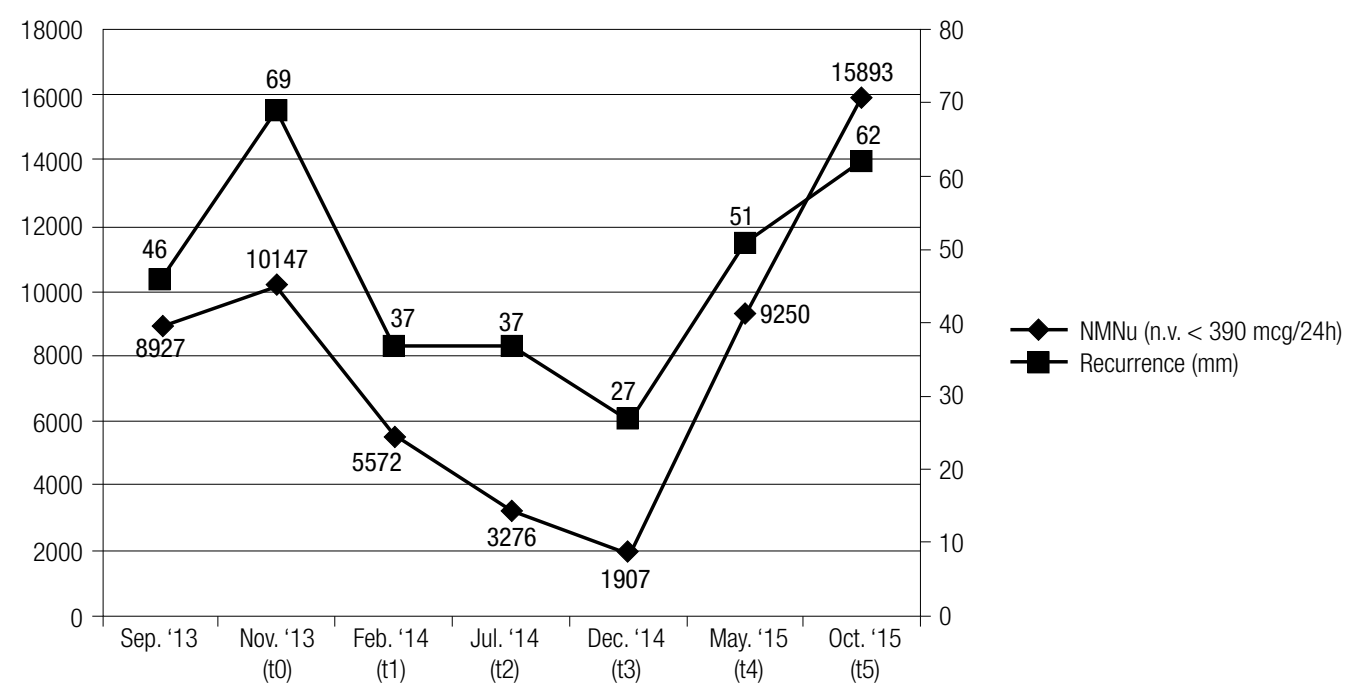

Figure 2. Trend of urinary normetanephrine (NMNu) combined with the size of tumor recurrence (mm).

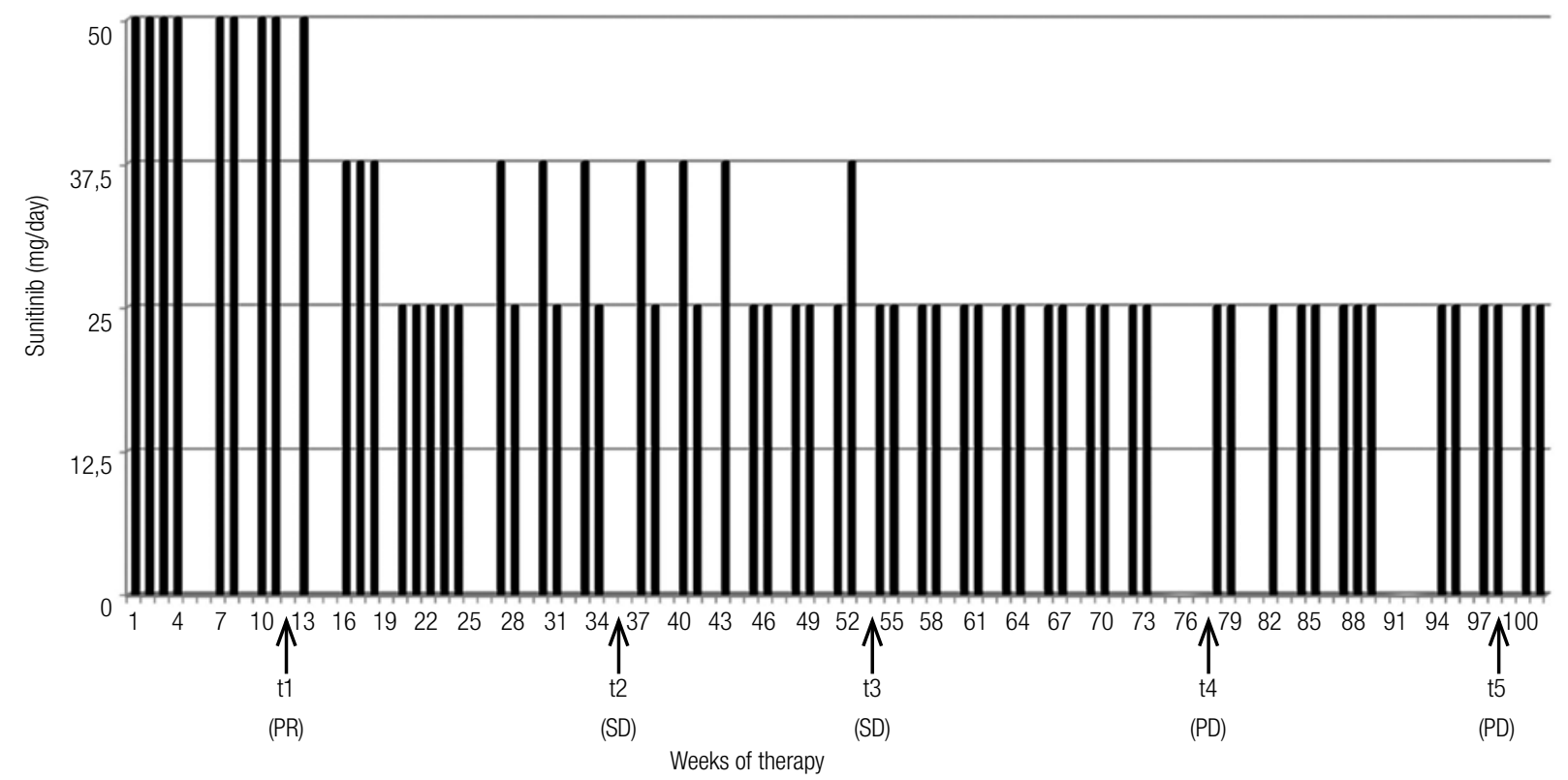

Figure 3. Therapy schedule from November 2013 (t0) to November 2015 (t5). 

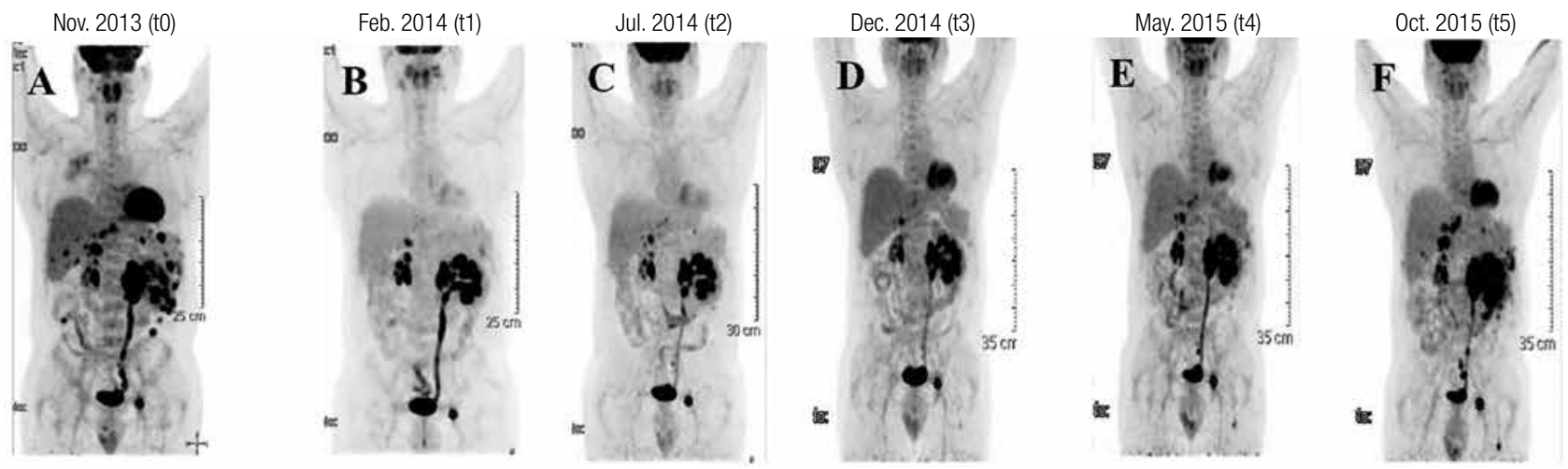

Figure 4. ${ }^{18} \mathrm{FDG}-\mathrm{PET}$ before $(\mathbf{A})$ and during (B-F) sunitinib therapy.

Table 1. Side effects evaluated by the Common Toxicity Criteria Manual version 2.0. Grade 0 no adverse event or within normal limits; grade 1 mild adverse event; grade 2 moderate adverse event; grade 3: severe and undesirable adverse event; grade 4 life-threatening or disabling adverse event; grade 5 death related to adverse event

\begin{tabular}{llc}
\hline Side effects & Drugs & Grades of adverse events \\
\hline Fatigue & - & 3 \\
Stomach pain with nausea and vomiting & Ranitidine and ondansetron & 3 \\
Hypothyroidism & Levothyroxine & 1 \\
Hypertriglyceridemia & $\omega 3$ and fenofibrate & 2 \\
Hypertension & Doxazosin, calcium antagonist & 2 \\
Sore mouth & Mouthwash with aloe or baking soda & 3 \\
\hline
\end{tabular}

\section{DISCUSSION}

In this paper we report the long lasting effect of sunitinib in a patient affected by a metastatic PGL. The surgical option on the primary lesion was discarded in view of the congenital right renal hypoplasia and the close adhesion of the tumor mass to the left kidney and ureter causing a high risk of postsurgical chronic renal failure. Radionuclide therapy with radiolabeled MIBG (15) or somatostatin analogs (16) was impeded by the insufficient uptakes of both the compounds. Therefore, we decided to start medical therapy using sunitinib.

Sunitinib is an oral tyrosine kinase inhibitor that targets the signaling pathways of VEGF receptors $l$ and 2 , PDGF- $\beta$ receptor, and other tyrosine kinases (c-KIT, FLT3, and RET) (17).

The therapy schedule recommended in the treatment of advanced renal cell carcinoma or gastrointestinal stromal tumors is $50 \mathrm{mg} /$ day 4 weeks on and 2 weeks off $(21)$, but it is generally accepted to adapt it to patient's tolerability. Therefore, in time, we reduced the doses and changed the drug schedule according to the side effects, mainly gastric pain and sore mouth, that the patient complained of. Thus, during the treatment, the patient's performance status was maintained grade 2 according to ECOG performance status criteria (18).

After an initial PD in the absence of therapy, Sunitinib caused a PR, as evaluated by RECIST criteria, lasting 3 months followed by a period of SD lasting 10 months.

In the following 12 months, we observed a slight progressive increase in the sizes of the primary lesion that nevertheless were still lower than those measured at the start of sunitinib administration ( $69 \mathrm{~mm}$ vs $62 \mathrm{~mm}$ ). As a whole, sunitinib slowed the disease progression in the last two years, allowing the patient to have a fairly good quality of life. It is also possible that the slight progression observed in the last 12 months might depend on the reduction in the drug doses, decided to limit its side effects.

To our knowledge, at present, only other 35 patients affected by a malignant PHEO/sPGL and treated with sunitinib have been reported in the literature (1213,18-28). Their characteristics, as well as those of our patient are reported in Table 2 . 
Table 2. Summary of the literature review.

\begin{tabular}{|c|c|c|c|c|c|c|c|}
\hline Author & $\begin{array}{l}\text { Age at the } \\
\text { time of } \\
\text { diagnosis }\end{array}$ & Tumor & $\begin{array}{l}\text { Genetic } \\
\text { analysis }\end{array}$ & $\begin{array}{l}\text { Surgery } \\
\text { before } \\
\text { sunitinib }\end{array}$ & Treatment & $\begin{array}{l}\text { Wk of } \\
\text { therapy }\end{array}$ & Outcome \\
\hline $\begin{array}{l}\text { Park KS and } \\
\text { cols., } 2009\end{array}$ & $\mathrm{M}(17 \mathrm{yr})$ & PHEO & NA & Yes & $\begin{array}{l}37,5 \mathrm{mg} / \text { day for } 7 \text { weeks and } 25 \mathrm{mg} / \\
\text { day for } 4 \text { weeks }\end{array}$ & 11 & $\begin{array}{l}\mathrm{PR}^{*} \text { after } 7 \text { weeks (according to } \\
{ }^{18} \mathrm{FDG} \text { uptake) followed by SD*} \\
\text { after } 11 \text { weeks }\end{array}$ \\
\hline $\begin{array}{l}\text { Jimenez } \mathrm{C} \text { and } \\
\text { cols., } 2009\end{array}$ & $F(32 y r)$ & $\begin{array}{l}\text { PHEO } \\
(10.5 \mathrm{~cm})\end{array}$ & VHL & Yes & $50 \mathrm{mg} /$ day 4 weeks on, 2 weeks off & 36 & $\mathrm{PR}^{\star}$ \\
\hline \multirow[t]{3}{*}{$\begin{array}{l}\text { Joshua AM and } \\
\text { cols., } 2009\end{array}$} & M (55 yr) & $\begin{array}{l}\text { Abdominal } \\
\text { PGL } \\
(14.4 \mathrm{~cm})\end{array}$ & SDHB & $\begin{array}{l}\text { No (after } \\
\text { six cycles) }\end{array}$ & $\begin{array}{l}\text { 50/mg day } 4 \text { weeks on, } 2 \text { weeks off } \\
\text { (before surgery); } 37.5 \mathrm{mg} / \text { day } 4 \text { weeks } \\
\text { on, } 2 \text { weeks off (after surgery) }\end{array}$ & 48 & $\begin{array}{l}\text { PR after } 36 \text { weeks followed by PD } \\
\text { after } 48 \text { weeks (+ surgery) }\end{array}$ \\
\hline & M (28 yr) & $\begin{array}{l}\text { Abdominal } \\
\text { PGL }(7 \mathrm{~cm})\end{array}$ & $\mathrm{SDHB}$ & Yes & 50/mg day 4 weeks on, 2 weeks off** & 40 & PR \\
\hline & $F(41 y r)$ & $\begin{array}{l}\text { PHEO } \\
(15 \mathrm{~cm})\end{array}$ & $\begin{array}{l}\text { Negative for } \\
\text { SDHB, } \\
\text { SDHD, RET } \\
\text { and VHL }\end{array}$ & Yes & $50 \mathrm{mg} /$ day 4 weeks on, 2 weeks off* & 40 & $\mathrm{PR}^{*}$ \\
\hline $\begin{array}{l}\text { Hahn NM and } \\
\text { cols., } 2009\end{array}$ & $F(33 y r)$ & $\begin{array}{l}\text { Abdominal } \\
\text { PGL }(17 \mathrm{~cm})\end{array}$ & SDHB & Yes & $\begin{array}{l}50 \mathrm{mg} / \text { day } 4 \text { weeks on, } 2 \text { weeks off; } \\
50 \mathrm{mg} / \text { day } 2 \text { weeks on, } 1 \text { week off }\end{array}$ & 16 & $P D^{*}$ \\
\hline Cirillo F, 2010 & M (37 yr) & $\begin{array}{l}\text { Abdominal } \\
\text { PGL } \\
(17 \times 14 \times 9 \mathrm{~cm})\end{array}$ & NA & Yes & $\begin{array}{l}50 \mathrm{mg} / \text { day } 4 \text { weeks on, } 2 \text { weeks off; } \\
25 \mathrm{mg} / \text { day } 4 \text { weeks on, } 2 \text { weeks off; } \\
25 \mathrm{mg} / \text { day } 2 \text { weeks on, } 1 \text { week off }\end{array}$ & 24 & $\begin{array}{l}\mathrm{SD}^{\star} \text { after } 15 \text { weeks followed by } \\
\mathrm{PD}^{\star} \text { after } 24 \text { weeks } \\
\text { (+ octreotide LAR) }\end{array}$ \\
\hline \multirow[t]{2}{*}{$\begin{array}{l}\text { Zukauskaite R } \\
\text { and cols., } 2011\end{array}$} & M (31 yr) & $\begin{array}{c}\text { PGL } \\
\text { thoracic- } \\
\text { lumbar region } \\
(10 \times 15 \mathrm{~cm})\end{array}$ & $\begin{array}{l}\text { No somatic } \\
\text { mutations }\end{array}$ & Yes & $50 \mathrm{mg} /$ day 4 weeks on, 2 weeks off & 24 & $\begin{array}{l}\mathrm{SD}^{*} \text { after } 12 \text { weeks followed by } \\
\mathrm{PD}^{\star} \text { after } 24 \text { weeks }\end{array}$ \\
\hline & $F(54 y r)$ & PHEO & Sporadic & Yes & $\begin{array}{l}50 \mathrm{mg} / \text { day } 4 \text { weeks on, } 2 \text { weeks off } \\
\text { reduced up to } 12.5 \mathrm{mg} / \text { day }\end{array}$ & 68 & $\begin{array}{l}\mathrm{SD}^{\star} \text { after } 40 \text { weeks followed by } \\
\mathrm{PD}^{\star} \text { after } 68 \text { weeks }\end{array}$ \\
\hline \multirow[t]{11}{*}{$\begin{array}{l}\text { Ayala-Ramirez M } \\
\text { and cols., } 2012 \text { F } \\
\text { (8); M (9) }\end{array}$} & $(33 \mathrm{yr})$ & PHEO & VHL & No & $\begin{array}{l}50 \mathrm{mg} / \text { day } 4 \text { weeks on, } 2 \text { weeks off or } \\
37.5 \mathrm{mg} / \text { day continously or } 37.5 \mathrm{mg} / \\
\text { day } 3 \text { weeks on, } 1 \text { week off }\end{array}$ & 24 & SD \\
\hline & $(60 \mathrm{yr})$ & PHEO & Sporadic & No & $\begin{array}{l}50 \mathrm{mg} / \text { day } 4 \text { weeks on, } 2 \text { weeks off or } \\
37.5 \mathrm{mg} / \text { day continously or } 37.5 \mathrm{mg} / \\
\text { day } 3 \text { weeks on, } 1 \text { week off }\end{array}$ & 44 & PR \\
\hline & (55 yr) & PGL & SDHB & No & $\begin{array}{l}50 \mathrm{mg} / \text { day } 4 \text { weeks on, } 2 \text { weeks off or } \\
37.5 \mathrm{mg} / \text { day continously or } 37.5 \mathrm{mg} / \\
\text { day } 3 \text { weeks on, } 1 \text { week off }\end{array}$ & 108 & SD \\
\hline & (20 yr) & PGL & SDHB & No & $\begin{array}{l}50 \mathrm{mg} / \text { day } 4 \text { weeks on, } 2 \text { weeks off or } \\
37.5 \mathrm{mg} / \text { day continously or } 37.5 \mathrm{mg} / \\
\text { day } 3 \text { weeks on, } 1 \text { week off }\end{array}$ & NA & SD \\
\hline & (62 yr) & PHEO & Sporadic & No & $\begin{array}{l}50 \mathrm{mg} / \text { day } 4 \text { weeks on, } 2 \text { weeks off or } \\
37.5 \mathrm{mg} / \text { day continously or } 37.5 \mathrm{mg} / \\
\text { day } 3 \text { weeks on, } 1 \text { week off }\end{array}$ & 1.6 & PD \\
\hline & (14 yr) & PHEO & Sporadic & No & $\begin{array}{l}50 \mathrm{mg} / \text { day } 4 \text { weeks on, } 2 \text { weeks off or } \\
37.5 \mathrm{mg} / \text { day continously or } 37.5 \mathrm{mg} / \\
\text { day } 3 \text { weeks on, } 1 \text { week off }\end{array}$ & 13 & PD \\
\hline & $(47 \mathrm{yr})$ & PHEO & Sporadic & No & $\begin{array}{l}50 \mathrm{mg} / \text { day } 4 \text { weeks on, } 2 \text { weeks off or } \\
37.5 \mathrm{mg} / \text { day continously or } 37.5 \mathrm{mg} / \\
\text { day } 3 \text { weeks on, } 1 \text { week off }\end{array}$ & 16 & $\mathrm{PD}$ \\
\hline & $(40 \mathrm{yr})$ & PHEO & Sporadic & No & $\begin{array}{l}50 \mathrm{mg} / \text { day } 4 \text { weeks on, } 2 \text { weeks off or } \\
37.5 \mathrm{mg} / \text { day continously or } 37.5 \mathrm{mg} / \\
\text { day } 3 \text { weeks on, } 1 \text { week off }\end{array}$ & 4 & PD \\
\hline & (57 yr) & PGL & SDHB & No & $\begin{array}{l}50 \mathrm{mg} / \text { day } 4 \text { weeks on, } 2 \text { weeks off or } \\
37.5 \mathrm{mg} / \text { day continously or } 37.5 \mathrm{mg} / \\
\text { day } 3 \text { weeks on, } 1 \text { week off }\end{array}$ & NA & $\begin{array}{l}\text { NA (sunitinib was stopped due to } \\
\text { toxicity) }\end{array}$ \\
\hline & (60 yr) & PGL & SDHB & No & $\begin{array}{l}50 \mathrm{mg} / \text { day } 4 \text { weeks on, } 2 \text { weeks off or } \\
37.5 \mathrm{mg} / \text { day continously or } 37.5 \mathrm{mg} / \\
\text { day } 3 \text { weeks on, } 1 \text { week off }\end{array}$ & NA & $\begin{array}{l}\text { NA (sunitinib was stopped due to } \\
\text { toxicity) }\end{array}$ \\
\hline & (69 yr) & PHEO & Sporadic & No & $\begin{array}{l}50 \mathrm{mg} / \text { day } 4 \text { weeks on, } 2 \text { weeks off or } \\
37.5 \mathrm{mg} / \text { day continously or } 37.5 \mathrm{mg} / \\
\text { day } 3 \text { weeks on, } 1 \text { week off }\end{array}$ & NA & $\begin{array}{l}\text { NA (sunitinib was stopped due to } \\
\text { toxicity) }\end{array}$ \\
\hline
\end{tabular}




\begin{tabular}{|c|c|c|c|c|c|c|c|}
\hline Author & $\begin{array}{l}\text { Age at the } \\
\text { time of } \\
\text { diagnosis }\end{array}$ & Tumor & $\begin{array}{l}\text { Genetic } \\
\text { analysis }\end{array}$ & $\begin{array}{l}\text { Surgery } \\
\text { before } \\
\text { sunitinib }\end{array}$ & Treatment & $\begin{array}{l}\text { Wk of } \\
\text { therapy }\end{array}$ & Outcome \\
\hline & (27 yr) & PHEO & SDHB & No & $\begin{array}{l}50 \mathrm{mg} / \text { day } 4 \text { weeks on, } 2 \text { weeks off or } \\
37.5 \mathrm{mg} / \text { day continously or } 37.5 \mathrm{mg} / \\
\text { day } 3 \text { weeks on, } 1 \text { week off }\end{array}$ & NA & SD \\
\hline & (56 yr) & PHEO & Sporadic & No & $\begin{array}{l}50 \mathrm{mg} / \text { day } 4 \text { weeks on, } 2 \text { weeks off or } \\
37.5 \mathrm{mg} / \text { day continously or } 37.5 \mathrm{mg} / \\
\text { day } 3 \text { weeks on, } 1 \text { week off }\end{array}$ & 48 & PR \\
\hline & $(45 \mathrm{yr})$ & PGL & SDHB & No & $\begin{array}{l}50 \mathrm{mg} / \text { day } 4 \text { weeks on, } 2 \text { weeks off or } \\
37.5 \mathrm{mg} / \text { day continously or } 37.5 \mathrm{mg} / \\
\text { day } 3 \text { weeks on, } 1 \text { week off }\end{array}$ & 18 & $\mathrm{PR}$ \\
\hline & $(40 \mathrm{yr})$ & PGL & SDHB & No & $\begin{array}{l}50 \mathrm{mg} / \text { day } 4 \text { weeks on, } 2 \text { weeks off or } \\
37.5 \mathrm{mg} / \text { day continously or } 37.5 \mathrm{mg} / \\
\text { day } 3 \text { weeks on, } 1 \text { week off }\end{array}$ & 32 & SD \\
\hline & $(43 \mathrm{yr})$ & PHEO & SDHB & No & $\begin{array}{l}50 \mathrm{mg} / \text { day } 4 \text { weeks on, } 2 \text { weeks off or } \\
37.5 \mathrm{mg} / \text { day continously or } 37.5 \mathrm{mg} / \\
\text { day } 3 \text { weeks on, } 1 \text { week off }\end{array}$ & 16.4 & PD \\
\hline & (63 yr) & PHEO & Sporadic & No & $\begin{array}{l}50 \mathrm{mg} / \text { day } 4 \text { weeks on, } 2 \text { weeks off or } \\
37.5 \mathrm{mg} / \text { day continously or } 37.5 \mathrm{mg} / \\
\text { day } 3 \text { weeks on, } 1 \text { week off }\end{array}$ & 8.4 & PD \\
\hline $\begin{array}{l}\text { Nemoto } \mathrm{K} \text { and } \\
\text { cols., } 2012\end{array}$ & $\mathrm{~F}(41 \mathrm{yr})$ & $\begin{array}{l}\text { PHEO } \\
(10 \mathrm{~cm})\end{array}$ & NA & Yes & $\begin{array}{l}50 \text { mg/day } 4 \text { weeks on, } 2 \text { weeks off; } 25 \\
\mathrm{mg} / \text { day } 2 \text { weeks on, } 2 \text { weeks off }\end{array}$ & 26 & $\mathrm{PR}$ \\
\hline \multirow[t]{3}{*}{$\begin{array}{l}\text { Sun FK and } \\
\text { cols., } 2012\end{array}$} & M (32 yr) & $\begin{array}{l}\text { PHEO } \\
(18 \mathrm{~cm})\end{array}$ & $\begin{array}{l}\text { Negative for } \\
\text { SDHB, } \\
\text { SDHD, RET } \\
\text { and VHL }\end{array}$ & Yes & $\begin{array}{l}50 \text { mg/day } 4 \text { weeks on, } 2 \text { weeks off; } \\
37.5 \text { mg/day } 4 \text { weeks on, } 2 \text { weeks off }\end{array}$ & 22 & $\begin{array}{l}\text { Enlargement in the necrosis area } \\
\text { of tumor with } \mathrm{SD}^{*}\end{array}$ \\
\hline & M (51 yr) & $\begin{array}{l}\text { PHEO } \\
(12.9 \mathrm{~cm})\end{array}$ & $\begin{array}{l}\text { Negative for } \\
\text { SDHB, } \\
\text { SDHD, RET } \\
\text { and VHL }\end{array}$ & Yes & $50 \mathrm{mg} /$ day 4 weeks on, 2 weeks off & 28 & $\begin{array}{l}\text { Necrosis of the lesions at the CT } \\
\text { scan }\left(\mathrm{PR}^{\star}\right)\end{array}$ \\
\hline & $F(49 \mathrm{yr})$ & $\begin{array}{l}\text { PHEO } \\
(5 \mathrm{~cm})\end{array}$ & $\begin{array}{l}\text { Negative for } \\
\text { SDHB, } \\
\text { SDHD, RET } \\
\text { and VHL }\end{array}$ & Yes & $50 \mathrm{mg} /$ day 4 weeks on, 2 weeks off & 30 & $\mathrm{PR}^{*}$ \\
\hline $\begin{array}{l}\text { Prochilo T and } \\
\text { cols., } 2012\end{array}$ & $\mathrm{~F}(35 \mathrm{yr})$ & $\begin{array}{l}\text { Abdominal } \\
\text { PGL }\end{array}$ & SDHB & Yes & $\begin{array}{l}50 \text { mg/day } 4 \text { weeks on, } 2 \text { weeks off; } \\
37.5 \mathrm{mg} \text { daily } 2 \text { weeks on, } 2 \text { weeks off; } \\
25 \mathrm{mg} \text { daily } 2 \text { weeks on, } 1 \text { week off }\end{array}$ & $\begin{array}{l}\text { More } \\
\text { than } 36\end{array}$ & $\begin{array}{l}\mathrm{PR}^{\star} \text { after } 12 \text { weeks followed by } \\
\mathrm{SD}^{\star} \text { after } 36 \text { weeks and finally } \\
\mathrm{PD}^{\star} \text { (evaluated by }{ }^{18} \mathrm{FDG}-\mathrm{PET} \text { ) }\end{array}$ \\
\hline \multirow[t]{2}{*}{$\begin{array}{l}\text { Hata J and cols., } \\
2014\end{array}$} & M (23 yr) & $\begin{array}{l}\text { PHEO } \\
(8.7 \mathrm{~cm})\end{array}$ & NA & Yes & 50 mg/day 4 weeks on, 2 weeks off & 20 & 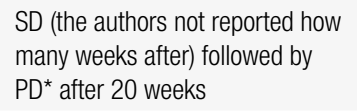 \\
\hline & $M(60 \mathrm{yr})$ & $\begin{array}{l}\text { PHEO } \\
(7.2 \mathrm{~cm})\end{array}$ & NA & Yes & 50 mg/day 4 weeks on, 2 weeks off** & 16 & $\begin{array}{l}\mathrm{SD}^{\star} \text { (the authors not reported how } \\
\text { many weeks after) followed by } \\
\mathrm{PD}^{\star} \text { after } 16 \text { weeks }\end{array}$ \\
\hline $\begin{array}{l}\text { Lebowitz-Amit R } \\
\text { and cols., } 2014\end{array}$ & M (51 yr) & $\begin{array}{l}\text { Abdominal } \\
\text { PGL }(6.9 \times 5.9 \\
\times 7.1 \mathrm{~cm})\end{array}$ & $\begin{array}{l}\text { Negative for } \\
\text { SDHB, } \\
\text { SDHC, } \\
\text { SDHD, } \\
\text { TMEM127 } \\
\text { and NF1 }\end{array}$ & Yes & $\begin{array}{l}50 \mathrm{mg} / \text { day; } 37.5 \mathrm{mg} / \mathrm{day} ; 25 / 37.5 \mathrm{mg} / \\
\text { day alternating }\end{array}$ & 24 & $\mathrm{SD}^{*}$ \\
\hline $\begin{array}{l}\text { Bourcier ME and } \\
\text { cols., } 2013\end{array}$ & $\mathrm{~F}(70 \mathrm{yr})$ & $\begin{array}{l}\text { Abdominal } \\
\text { PGL }\end{array}$ & NA & Yes & $50 \mathrm{mg} /$ day 4 weeks on, 2 weeks off & 12 & $\mathrm{CR}$ \\
\hline Our case & M (35yr) & $\begin{array}{l}\text { Abdominal } \\
\text { PGL }\end{array}$ & SDHB & yes & $\begin{array}{l}25 \mathrm{mg} / \text { day } 2 \text { weeks on, } 1 \text { week off for } \\
\text { the of time }\end{array}$ & 101 & $\begin{array}{l}\text { PR after } 12 \text { weeks followed by SD } \\
\text { after } 32 \text { weeks up to } 54 \text { weeks } \\
\text { and PD after } 78 \text { weeks }\end{array}$ \\
\hline
\end{tabular}

F: female; M: male; NA: not available; PGL: paraganglioma; PHEO: pheochromocytoma; * data deducted by the case description and not by RECIST evaluation; ** deducted data. Weeks are always reported from the beginning of sunitinib therapy.

In this series 20 patients were males and 16 females, aging from 14 to 70 yrs.
Genetic analysis was performed in 30 out of 36 patients. 15 patients resulted wild-type, 2 Von Hippel 
Lindau (VHL) mutation carriers and $13 S D H B$ mutation carriers. Therefore, a $S D H B$ germ-line mutation was found in $43.3 \%(13 / 30)$ of genotyped patients, in agreement with the high frequency of malignancy reported in $S D H B$ mutation carriers (29).

Surgery on the primary tumor had been carried out in $50 \%$ of patients $(18 / 36)$. Sunitinib was administered as first non surgical therapy in $33 \%(12 / 36)$.

The length of sunitinib therapy has been reported in $31 / 36$ patients and ranges from 16 to 108 weeks.

The outcome of sunitinib therapy has been reported according to different criteria: in 19 patients the outcome has been calculated by RECIST criteria while in 14 patients the outcome has been reported by unspecified criteria. In 3 patients the outcome was not reported as the treatment was interrupted shortly after the start of therapy because of drug toxicity.

As a whole, 7 patients experienced PD, while sunitinib resulted effective in $72.2 \%$ of patients $(26 / 36)$, providing a complete response (CR) in 1 patient, a PR in 13 patients and a SD in 12 patients.

CR was observed after 12 weeks. In all patients $(13 / 13)$ with PR the length of therapy is reported and the drug effect was maintained after a period ranging from 11 to 101 weeks. In this group, PD latterly ensued in 3 patients, after 48 , more than 36 and 78 weeks from the beginning of therapy.

Among the 12 patients found with SD the length of therapy was reported in ten of them, ranging from 16 to 108 weeks and 5 were reported to undergo PD at different times after the beginning of therapy (from 16 to 68 weeks).

It has been hypothesized that the genetic background might affect the effects of antiangiogenic therapy, resulting more effective in $\mathrm{PHEO} / \mathrm{PGL}$ belonging to cluster l (30).

When analyzing this series reported in the literature, among the 30 genotyped patients, the outcome was reported in 14 wild type (wt) patients and in 13 mutation carriers (11 SDHB and 2 VHL). PR or SD was reported in $9 \mathrm{wt}$ and 11 mutated patients, respectively. Therefore, at least from this limited series, the different genetic background does not seem to affect sunitinib efficacy.

In summary, from the scant data of the literature, sunitinib seems able, in some patients, to slow the progression of the disease and its efficacy does not seem to depend on tumor genetic background.
Nevertheless, its real efficacy will be stated only after the results of proper controlled studies. At present, only two such studies are ongoing in patients with malignant PHEOs/sPGLs: the FIRSTMAPPP study (First International Randomized Study in Malignant Progressive Pheochromocytoma and Paraganglioma) and the SNIPP study (Study of Sunitinib in Patients with Recurrent Paraganglioma/Pheochromocytoma). Both are Phase II studies.

FIRSTMAPPP is a multicenter and randomized study (sunitinib $37.5 \mathrm{mg} /$ day versus placebo) while SNIPP is a non randomized study (sunitinib $50 \mathrm{mg}$ / day 4 weeks on, 2 weeks off).

In conclusion, we describe the case of a patient with malignant PHEO treated only with sunitinib for a very long period (101 weeks). The drug was able to induce a PR after 12 weeks and to maintain its effects (SD) for an additional 66 weeks. In spite of a slow disease progression, at present the patient still presents a good quality of life. Because of PD, the patient has started treatment with Temozolomide.

The results we observed in our patient are similar to those reported in other patients similarly affected by malignant PHEO/PGL. As a whole, sunitinib seems to offer a therapeutic option in some of these patients, although its effect seems limited in time.

Acknowledgements: funding support for this paper has been obtained by the University of Florence and by Paradifference Foundation. Letizia Canu, Elena Rapizzi, Rossella Fucci, Tonino Ercolino and Massimo Mannelli are members of the ENS@T (European Network for the Study of Adrenal Tumors).

Disclosure: no potential conflict of interest relevant to this article was reported.

\section{REFERENCES}

1. Dahia PL. Pheochromocytoma and paraganglioma pathogenesis: learning from genetic heterogeneity. Nat Rev Cancer. 2015;14:108-19.

2. Gimenez-Roqueplo $A P$, Favier J, Rustin P, Rieubland C, Crespin $\mathrm{M}, \mathrm{Nau} \mathrm{V}$, et al. Mutations in the SDHB gene are associated with extra-adrenal and/or malignant phaeochromocytomas. Cancer Research. 2003;63:5615-21.

3. Lenders JWM, Eisenhofer G, Mannelli M, Pacak K. Phaeochromocytoma. Lancet. 2005;366:665-75.

4. Adjalle R, Plouin PF, Pacak K, Lehnert H. Treatment of malignant pheochromocytoma. Horm Metab Res. 2009;41:687-96.

5. John H, Ziegler WH, Hauri D, Jaeger P. Pheochromocytomas: can malignant potential be predicted? Urology. 1999;53:679-83.

6. Eisenhofer G, Bornstein SR, Brouwers FM, Cheung Nk, Dahia $P L$, de Krijger RR, et al. Malignant pheochromocytoma: current status and initiatives for future progress. Endocr Relat Cancer. 2004;11:423-36. 
7. Timmers HJ, Gimenez-Roqueplo AP, Mannelli M, Pacak K. Clinical aspects of SDHx-related pheochromocytoma and paraganglioma. Endocr Relat Cancer. 2009;16:391-400.

8. Andersen KF, Altaf R, Krarup-Hansen A, Kromann-Andersen B, Horn T, Christensen NJ, et al. Malignant pheochromocytomas and paragangliomas - The importance of a multidisciplinary approach. CancerTreat Rev. 2011;37:111-9.

9. Teunissen JJ, Kwekkeboom DJ, Valkema R, Krenning EP. Nuclear medicine techniques for the imaging and treatment of neuroendocrine tumours. Endocr Relat Cancer. 2011;18 Suppl 1:S27-51.

10. Huang $H$, Abraham J, Hung E, Averbuch S, Merino M, Steinberg SM et al. Treatment of malignant pheochromocytoma/ paraganglioma with cyclophosphamide, vincristine, and dacarbazine: recommendation from a 22-year follow-up of 18 patients. Cancer. 2008;113:2020-8.

11. Ekeblad S, Sundin A, Janson ET, Welin S, Granberg D, Kindmark $\mathrm{H}$, et al. Temozolomide as monotherapy is effective in treatment of advanced malignant neuroendocrine tumors. Clin Cancer Res. 2007;13:2986-91.

12. Joshua AM, Ezzat $S$, Asa SL, Evans A, Broom R, Freeman M, et al. Rationale and evidence for sunitinib in the treatment of malignant paraganglioma/pheochromocytoma. J Clin Endocrinol Metab. 2009;94:5-9.

13. Park KS, Lee JL, Ahn H, Koh JM, Park I, Choi JS, et al. Sunitinib, a novel therapy for anthracycline- and cisplatin-refractory malignant pheochromocytoma. JpnJ Clin Oncol. 2009;39:327-31.

14. Eisenhauer EA, Therasse P, Bogaerts J, Schwartz LH, Sargent D, Ford $R$, et al. New response evaluation criteria in solid tumours: Revised RECIST guideline (version 1.1). Eur J Cancer. 2009;45:228-47.

15. Hartley A, Spooner D, Brunt AM. Management of malignant phaeochromocytoma: a retrospective review of the use of MIBG and chemotherapy in the West Midlands. Clin Oncol (R Coll Radiol). 2001;13:361-6.

16. Kopf D, Bockisch A, Steinert H, Hahn K, Beyer J, Neumann HP, et al. Octreotide scintigraphy and catecholamine response to an octreotide challenge in malignant phaeochromocytoma. Clin Endocrinol (Oxf). 1997;46:39-44.

17. Jimenez C, Cabanillas ME, Santarpia L, Jonasch E, Kyle KL, Lano EA, et al. Use of the Tyrosine Kinase Inhibitor Sunitinib in a Patient with von Hippel-Lindau Disease: Targeting Angiogenic Factors in Pheochromocytoma and Other von Hippel-Lindau DiseaseRelatedTumors. J Clin Endocrinol Metab. 2009;94:386-91.

18. Oken MM, Creech RH, Tormey DC, Horton J, Davis TE, McFadden ET, et al. Toxicity and response criteria of the Eastern Cooperative Oncology Group. Am J Clin Oncol. 1982;5:649-55.
19. Hahn NM, Reckova $M$, Cheng $L$, Baldridge LA, Cummings OW, Sweeney CJ. Patient with malignant paraganglioma responding to the multikinase inhibitor sunitinib malate. J Clin Oncol. 2009;27:460-3.

20. Cirillo F. Metastatic paraganglioma and treatment with sunitinib: a case report. Tumori. 2010;96:1022-7.

21. Zukauskaite R, Hjorthgau K, Poulsen PL, Baerentzen S, Ladekarl $M$. Malignant pheochromocytoma and paraganglioma: three cases illustrating the use of molecular targeted diagnostics and therapy and possible role of new drugs. Acta Oncol. 2011;50(8):1255-9.

22. Ayala-Ramirez $M$, Chougnet $C N$, Habra MA, Palmer JL, Leboulleux S, Cabanillas ME, et al. Treatment with Sunitinib for Patients with Progressive Metastatic Pheochromocytomas and Sympathetic Paragangliomas. J Clin Endocrinol Metab. 2012;97(11):4040-50.

23. Nemoto K, Miura T, Shioji G, Tsuboi N. Sunitinib treatment for refractory malignant pheochromocytoma. Neuro Endocrinol Lett. 2012;33(3):260-4

24. Sun FK, He HC, Su TW, Zhou WL, Huang X, Dai J, et al. Multitargeted tyrosine kinase inhibitor sunitinib: a novel strategy for sporadic malignant pheochromocytoma. Chin Med J (Engl). 2012;125:2231-4.

25. Prochilo T, Savelli G, Bertocchi P, Abeni C, Rota L, Rizzi A, et al. Targeting VEGF-VEGFR Pathway by Sunitinib in Peripheral Primitive NeuroectodermalTumor, Paraganglioma and Epithelioid Hemangioendothelioma: Three Case Reports. Case Rep Oncol. 2012;6:90-7.

26. Hata J, Haga N, Ishibashi K, Takahashi N, Ogawa S, Kataoka M, et al. Sunitinib for refractory malignant pheochromocytoma: two case reports. Int Urol Nephrol. 2014;46:1309-12.

27. Lebowitz-Amit R, Mete O, Asa SL, Ezzat S, Joshua AM. Malignant pheochromocytoma secreting vasoactive intestinal peptide and response to sunitinib: a case report and literature review. Endocr Pract. 2014;20:e145-50.

28. Bourcier ME, Vinik Al. Sunitinib for the treatment of metastatic paraganglioma and vasoactive intestinal polypeptide-producing tumor (VIPoma). Pancreas. 2013;42:348-52.

29. Gimenez-Roqueplo AP, Burnichon N, Amar L, Favier J, Jeunemaitre X, Plouin PF. Recent advances in the genetics of pheochromocytoma and functional paraganglioma. Clin Exp Pharmacol Physiol. 2008;35:376-9.

30. Santarpia L, Habra MA, Jimenez C. Malignant pheochromocytomas and paragangliomas: molecular signaling pathways and emerging therapies. Horm Metab Res. 2009;41:680-6. 